\title{
El Valor Pedagógico del Aula Virtual: Hablan los usuarios
}

\author{
The Pedagogical Value of the Virtual Classroom (VC): the users talk
}

\author{
Lea Sulmont Haak
}

\begin{abstract}
Resumen
¿Cuál es el valor pedagógico que tiene el Aula Virtual para los alumnos y docentes? El siguiente trabajo presenta los resultados de un estudio longitudinal sobre la integración del Aula Virtual (AV) en la universidad. Se analiza, desde una perspectiva comprensiva, los propósitos de uso del AV en la un espacio formal y presencial de aprendizaje, como es caso de la Universidad Peruana de Ciencias Aplicadas (UPC). Se plantea reconocer su valor pedagógico; es decir, su utilidad para los usuarios como herramienta de apoyo en su proceso de enseñanza-aprendizaje. Este valor es reconocido desde la experiencia concreta del uso en un contexto de aprendizaje. Para ello, el estudio se estructura en cuatro niveles de análisis complementarios: la evolución del uso del AV en la UPC, la percepción sobre el uso de Tecnologías de la Información y de la Comunicación (TICs) en la educación, los propósitos del empleo del AV y la percepción de su valor pedagógico. Finalmente, se comenta cómo el AV es una herramienta que amplifica la práctica pedagógica; considerando, sin embargo, que su sola presencia no implica necesariamente una evolución de esta práctica, ya que se exige también la renovación de las competencias de los usuarios.
\end{abstract}

Palabras clave: Entorno Virtual de aprendizaje (AV), Universidad, TICs, valor pedagógico.

\begin{abstract}
Abastract
What is the pedagogical value of the Virtual Classroom for the students and for the teachers? This work presents the results of a longitudinal study about the integration of the Virtual Classroom at the university and analyzes, from a comprehensive perspective, the types of uses of the VC in a formal learning environment like at the University, where the presence of the teacher is a must. It sets forth the concept of the pedagogical value understood as the benefit the users perceive from this tool as a means of support in their teaching-learning process. This value can only be recognized when it is used in a concrete experience within a learning situation. For this, the study is structured in four levels of complementary analysis: the evolution of the use of the VC at the UPC, the perception of the use of the ITC's (Information Technology and Communication) in education, the purposes of the uses of the VC and the perception of the pedagogical value of the VC. Finally, the article discusses how the Virtual Classroom amplifies the pedagogical practice and declares that the sole presence of such technological tool does not necessarily mean an evolution in these practices. Without doubt, the article demands for the renovation of the users' capabilities.
\end{abstract}

Key words: Virtual Classroom (Virtual Environment in the learning process), ITC's (Information Technology and Communication), pedagogical value.. 
Аก๊̃ 3- N²-Dic. 2007

\section{Introducción}

“Quizás fue este el ciclo donde descubrimos que el Aula Virtual tenía tantos usos y tantas herramientas útiles para poder aprender cada vez más. Gracias al AV, hemos podido hacer trabajos de forma más sincronizada, solucionar dudas en el foro, acceder a información desde cualquier parte (lo que permite que estemos más preparados antes de la clase), entre otras que han hecho que nuestro rendimiento sea mayor”.

Alumno del Curso SI118, Relato de Experiencia 2006-1.

Cuatro años han transcurrido desde que se inició el proceso de integración del Aula Virtual (AV)1 en la Universidad Peruana de Ciencias Aplicadas (UPC), por ello consideramos oportuno reflexionar sobre el valor de la introducción de esta herramienta tecnológica en las formas y espacios de interacción entre docentes y alumnos. Esta reflexión permitirá también repensar los procesos educativos como procesos más amplios, con nuevos contextos, escenarios y roles, en los que las tecnologías asumen un papel protagónico.

El AV se ha convertido en una herramienta cada vez más presente en los procesos formativos. En la actualidad, son cada vez más las instituciones educativas que implantan estas soluciones ${ }^{2}$. Por ejemplo, en la educación superior el empleo de ambientes virtuales de aprendizaje se ha generalizado, dejando de ser un patrón exclusivo de las universidades abiertas y a distancia o de las universidades virtuales. Se han convertido en herramientas que las universidades tradicionales integran a sus actividades formativas.

Técnicamente, el AV es un sistema de software de gestión del aprendizaje que sintetiza la funcionalidad del software de comunicación mediada por computadora (correo electrónico, tablones de anuncios, foros, chat) y métodos virtuales de envío de materiales de aprendizaje.

En la práctica, el AV del curso posibilita la gestión de actividades académicas en comunidades virtuales cerradas, donde es posible dictar una clase, trabajar un foro, intercambiar archivos, y comunicarse ya sea de forma sincrónica o asíncrona.

\footnotetext{
${ }^{1}$ En adelante emplearemos la abreviación AV para referirnos al Aula Virtual.

2 Thot, Nouvelles de la formatioon â distance (http://thot.cursus.edu/) tiene un repertorio de más de 163 herramientas empleadas como entornos virtuales de aprendizaje.
} 
La combinación de elementos tecnológicos al interior de un contexto educativo globalizado origina el desarrollo de una fuerte tendencia de virtualización de las actividades formativas; en el caso del contexto universitario, deviene en la incorporación de un entorno virtual como complemento a la enseñanza y aprendizaje tradicional. Lo importante es entender que, aunque la tecnología es el principal factor detrás de la tendencia de virtualización de la universidad (Tschang, 2001; Della Senta, 2003; Bates, 2001), no es la pieza central. El asunto no se limita a la inserción de las TICs; compromete también al conjunto de estructuras de la institución, que debe hacer frente a esta evolución con flexibilidad, de modo que pueda responder a las necesidades de formación continua y logre adaptarse a los espacios, tiempos y ritmos de aprendizaje. De este modo se consigue generar conocimiento con la potencia de las nuevas tecnológicas (LMS, CMS, etc.). Asimismo, implica integrar los recursos tecnológicos para apoyar eficiente y efectivamente los procesos de gestión académicoadministrativos, así como las gestiones del conocimiento y del proceso de enseñanzaaprendizaje. Finalmente, cada institución debe potenciar su identidad. Por ello, no existe un único modelo o patrón de universidad virtual. Lo que existe son propuestas híbridas que se van construyendo en el tiempo y en las dinámicas propias de los seres que conforman una comunidad particular ${ }^{3}$.

\section{La integración del Aula Virtual (AV) en la propuesta educativa de la Universidad Peruana de Ciencias Aplicadas}

Siguiendo a Bates ${ }^{4}$, el proceso de integración de tecnologías en la UPC ha seguido el modelo de planeamiento estratégico a nivel institucional. Desde el año 2004, la UPC ha incorporado a las TICs como parte de su propuesta educativa, implementado políticas y estrategias para su integración. El eje común de estas iniciativas ha sido potenciar el Modelo Educativo UPC, proporcionando herramientas tecnológicas que aporten a la calidad del proceso de enseñanza-aprendizaje.

La experiencia de implementación del AV en la UPC forma parte de un proyecto institucional $^{5}$ en el cual se diseñan servicios integrados y estrategias de apoyo a la gestión del conocimiento y del proceso de enseñanza-aprendizaje. El AV se incorporó para todos los cursos del Pregrado regular (AC) en el ciclo académico 2004-01 (811 cursos). A partir de 2005, la herramienta se introdujo en la División de Estudios para Ejecutivos (EPE), una nueva modalidad de Pregrado, cuyo público está conformado por

\footnotetext{
${ }^{3}$ Cfr. Sulmont 2004: 164.

${ }^{4}$ Cfr. Bates 2001: 92

${ }^{5}$ Proyecto Ágora.
} 
el adulto que trabaja y que sigue una formación en horario nocturno. A nivel operativo, la Dirección de Sistemas de la UPC es el área responsable del soporte tecnológico, mientras la coordinación pedagógica de proyectos de Tecnologías de la Información y de la Comunicación en la Educación es asumida por TICE, un área creada en 2004 como parte del Departamento de Calidad Educativa.

El AV es una pieza fundamental que evoluciona y se promueve como parte de la implementación del modelo pedagógico en la universidad. Por ello, a nivel organizacional, las TICs se integran sistemáticamente a la organización a partir de la perspectiva de la ingeniería de la formación ${ }^{6}$. Concebidas como artefactos, resultan poderosos amplificadores de las prácticas educativas. En tal sentido, existe la necesidad de orientar estas prácticas en la dirección del Modelo Educativo que propone la UPC, por lo que las TICs no son simples medios para el estudio, sino "artefactos" que condicionan o influyen en la creación de contextos específicos de enseñanzaaprendizaje” (Badia 2006:1).

El AV en la UPC no es solo un espacio virtual de apoyo al proceso de enseñanzaaprendizaje presencial, con herramientas que permiten el intercambio de información y la comunicación entre alumnos y profesores. También es un punto de encuentro de las diferentes áreas y actores comprometidos en la mejora e innovación de los procesos de enseñanza-aprendizaje.

Desde el punto de vista del aprendizaje, la integración de las TICs, sintetizadas en el uso de un AV, se apoya en el encuentro de una visión cognitiva del mismo, poniendo énfasis en los procesos mentales del sujeto que aprende. Asimismo, asume una postura social del conocimiento, analizando los procesos de aprendizaje e interacción entre pares y docentes, en un espacio contextualizado.

Para analizar los distintos tipos de uso dados al AV en la UPC, resulta inspiradora la propuesta de Badia ${ }^{7}$ sobre las ayudas educativas que brindan las TICs, enumerando seis finalidades de uso de la herramienta. A saber:

- Motivación, en tanto ofrece situaciones o contenidos que conectan al alumno con una actividad de aprendizaje y un contexto determinado.

\footnotetext{
${ }^{6}$ Cfr. Sulmont 2004: ¿PÁG?
}

${ }^{7}$ Cfr. Badia 2005: ¿PÁG? 
Aก̃o 3 - №2- Dic. 2007

- Organización del estudio, en tanto ofrece herramientas e información para favorecer la planificación de las actividades de aprendizaje (sílabo, cronograma, calendarios, agendas, etc.).

- Apoyo a la provisión de contenidos, en tanto facilita la distribución de los materiales del curso (así como recursos complementarios), garantizando una disponibilidad de los mismos en cualquier momento y desde cualquier lugar.

- Apoyo a la construcción del conocimiento, en tanto permite la realización de actividades directamente involucradas con el aprendizaje, como la búsqueda de información, la organización de actividades, la producción y envío de tareas, el trabajo en equipo, etc.

- Comunicación, en tanto promueve la interacción entre pares y docentes de manera sincrónica y asincrónica, favoreciendo el intercambio, la discusión y la colaboración.

- $\quad$ Seguimiento y evaluación, en tanto permite rastrear las actividades del alumno, monitorear su avance y evaluar el progreso del aprendizaje.

En el transcurso de la integración de las TICs a la educación, nos hemos preguntado con insistencia qué piensan los usuarios de las tecnologías y cuál es el valor pedagógico que perciben. A nivel institucional, esta reflexión implica evaluar si el uso del AV está teniendo un impacto que trascienda las experiencias individuales. Es decir, a nivel del modelo educativo.

\section{Metodología}

Para comprender y evaluar la evolución del uso del AV, se integran diversos tipos de fuentes. Por un lado, las fuentes cuantitativas, que provienen del monitoreo regular de las visitas sobre la base de los siguientes indicadores: cuánto, cuándo, por quiénes y cómo ha sido usada el AV. Las fuentes cualitativas, por su parte, son producto de los comentarios de alumnos y docentes en los focus group organizados por el Departamento de Calidad Educativa como estrategia de evaluación continua.

Adicionalmente, como parte de las estrategias de difusión de la herramienta, anualmente se organiza el concurso "Conectados al Aula Virtual”, cuyo objetivo es promover su uso reconociendo al grupo de profesores y alumnos que más la empleó durante el ciclo académico. El concurso también promueve la reflexión de los docentes sobre las prácticas pedagógicas que el AV permite innovar. En las tres primeras ediciones se han recogido valiosos relatos de alumnos y profesores que rinden testimonio de su experiencia con la herramienta. 
Finalmente, en 2006 se realizó una encuesta para estudiar la percepción de los alumnos y docentes sobre el uso de las TICs (en particular, el AV) en la educación. Dicha investigación fue producto de un encuentro del área de TICE de la UPC (Perú) y el Instituto de Pedagogía Multimedia (IPM)8 de la Universidad Católica de Lovaina (Bélgica). Entre los principales objetivos del estudio, se plantearon el reconocimiento de los aspectos motivacionales que influyen en el uso de tecnologías en la educación, así como las condiciones y finalidad de uso del AV. También se planteó estudiar la percepción de los usuarios sobre el valor pedagógico de esta herramienta.

A fines del año académico 2005-2006, el IPM aplicó una encuesta para evaluar el valor pedagógico de su plataforma icampus9. Luego de adaptaciones realizadas por el área de TICE, la encuesta fue replicada por la UPC en diciembre de 2006 por muestreo al 20\% de la población de estudiantes ( $\mathrm{AC}=1141 ; \mathrm{EPE}=510)$ y al $10 \%$ de los docentes $(\mathrm{AC}=62$; $E P E=25)$. El perfil de los encuestados fue representativo del universo estudiado, tanto por la distribución de género como de edad.

Además del valor comparativo del estudio, los resultados del mismo han sido de gran valor para las áreas dedicadas a la promoción de las TICs en la educación.

\section{El Valor pedagógico del Aula Virtual}

Entendemos por valor pedagógico del AV la utilidad que perciben los usuarios de la herramienta como apoyo a su proceso de enseñanza-aprendizaje. Si bien este valor tiene un componente subjetivo -pues se basa en la percepción de los individuos respecto a su experiencia concreta de uso-, consideramos que es posible aproximarse a esta noción, integrando los datos cuantitativos del empleo de la herramienta con los comentarios de los usuarios. Por tal motivo, a continuación presentamos cuatro niveles de análisis complementarios:

1. Evolución del uso del AV en la UPC.

2. Percepción sobre el uso de TICs en la educación.

3. Propósito de empleo del AV.

4. Percepción del valor pedagógico.

\footnotetext{
${ }^{8}$ ( http://www.ipm.ucl.ac.be/)

${ }^{9}$ (http://www.icampus.ucl.ac.be/)
} 
Aก̃̃ 3 - N²- Dic. 2007

\section{Evolución del uso del AV en la UPC}

El análisis histórico del uso del AV demuestra un crecimiento continuo desde su implantación. Sin embargo, el indicador que refleja con mayor nitidez la paulatina inserción del AV en el Pregrado es el incremento del porcentaje de Aulas Virtuales Usadas $^{10 .}$

Tabla No 1 Evolución histórica del uso del AV en el Pregrado UPC (2004-1 /2007-0)

\begin{tabular}{|l|c|c|c|c|c|c|c|c|c|}
\hline & $\mathbf{2 0 0 4 - 1}$ & $\mathbf{2 0 0 4 - 2}$ & $\mathbf{2 0 0 5 - 0}$ & $\mathbf{2 0 0 5 - 1}$ & $\mathbf{2 0 0 5 - 2}$ & $\mathbf{2 0 0 6 - 0}$ & $\mathbf{2 0 0 6 - 1}$ & $\mathbf{2 0 0 6 - 2}$ & $\mathbf{2 0 0 7 - 0}$ \\
\hline $\begin{array}{l}\text { Visitas totales } \\
\text { (alumnos y } \\
\text { docentes) }\end{array}$ & 13600 & 56656 & 10197 & 205277 & 189161 & 16682 & 359570 & 377925 & 41689 \\
\hline Aulas Usadas* & $4.3 \%$ & $21.2 \%$ & $14.7 \%$ & $59.9 \%$ & $58 \%$ & $51 \%$ & $71 \%$ & $72 \%$ & $68.4 \%$ \\
\hline $\begin{array}{l}\text { Población de } \\
\text { alumnos }\end{array}$ & 3920 & 2620 & 1826 & 4735 & 4527 & 1802 & 5671 & 5443 & 2609 \\
\hline $\begin{array}{l}\text { Población de } \\
\text { docentes }\end{array}$ & 719 & 769 & 176 & 653 & 672 & 133 & 732 & 696 & 176 \\
\hline Cursos/secciones & 580 & 572 & 170 & 943 & 934 & 138 & 1099 & 1058 & 187 \\
\hline
\end{tabular}

* Porcentaje de Aulas Usadas vs. Aulas Creadas. Corte en 80 visitas.

Fuente: Informe TICE, UPC (Marzo, 2007)

Si bien el crecimiento del uso del AV ha sido continuo, identificamos que no ha resultado homogéneo. La comparación de indicadores debe tomar en cuenta las particularidades de cada ciclo respecto a su duración, población y número de cursossección. Por ejemplo, el ciclo cero (que se desarrolla durante las vacaciones de verano) tiene una duración de 8 semanas en lugar de las 17 de los ciclos regulares (1 y 2). De otro lado, históricamente el primer ciclo tiene una mayor población de alumnos y cursos respecto al segundo. Tomando en cuenta estas consideraciones, el pico de crecimiento más importante se produjo durante el primer año de implantación del AV, entre el ciclo 2004-1 y el 2005-1, en el cual el número de visitas al AV creció en un 93\%.

\footnotetext{
${ }^{10}$ El indicador de Aulas Virtuales Usadas nos permite distinguir las AV que han tenido un uso en el ciclo de aquellas que no han tenido un empleo significativo. Se calcula tomando en cuenta solo aquellas AV que registran más de ochenta visitas o inicios de sesión por ciclo. Sobre esta base se calcula el porcentaje que representan estas Aulas frente al total de Aulas Creadas.
} 
Un aspecto destacable es que, si bien la población del Pregrado aumentó en 36\% entre los ciclos 2004-1 y 2006-2, el incremento de visitas al AV en aquel período fue ampliamente superior (2679\%). Asimismo, las Aulas Usadas crecieron en un 69\% en términos relativos o porcentuales.

A fines del año 2006, el 72\% de los cursos del Pregrado de la UPC, así como el 80\% de los cursos de EPE, empleaba el AV como herramienta de apoyo al proceso de enseñanza-aprendizaje.

Este importante incremento de las visitas al AV respecto al crecimiento demográfico de la población de usuarios, nos permite reconocer una dinámica de paulatina inserción del AV a las prácticas de alumnos y docentes. Esta tendencia refleja, en cierto modo, la utilidad que la herramienta tiene para los usuarios, dado que su uso es cada vez más frecuente e intenso.

\section{Percepción sobre el uso de TICs en la educación}

Como parte del intercambio (IPM-TICE), se propuso analizar la percepción de los usuarios respecto a los factores que representaban facilidades y obstáculos en el uso de TICs en la educación.Estos factores fueron determinados por los resultados de una encuesta abierta a los usuarios, en la cual se les solicitaba explicar las razones por las que utilizaban las TICs en su actividad académica, así como los motivos que limitaban su uso. La escala de respuesta seleccionada incluyó cuatro opciones: totalmente en desacuerdo, en desacuerdo, de acuerdo, y totalmente de acuerdo. La valoración de estos factores (9 facilidades y 9 obstáculos) resultó interesante. Por un lado, permitió comprender cómo las diferentes generaciones de alumnos y docentes percibían el uso de estas herramientas en la educación. Por otro lado, señalaban estrategias para la formación y difusión de las TICs en la educación.

\subsection{Facilidades para el uso de TICs en la Educación}

En promedio, el 81,5\% de los alumnos encuestados manifestó estar de acuerdo con las proposiciones sugeridas como facilidades para el uso de TICs en la educación $(\mathrm{AC}=79,5 \%$; $\mathrm{EPE}=83,5 \%)$.

En el Pregrado (AC), la proposición que resaltó por su mayor grado de acuerdo (82,2\%) fue "Las TICs me permiten hacer mejores trabajos y presentaciones". Por el contrario, el mayor desacuerdo (37.87\%) le fue otorgado a la frase "Los materiales en la Web son más fáciles de estudiar que los materiales impresos". Esta disconformidad fue significativamente mayor (>7\%) en el caso de las mujeres. 
Аก̃ם 3 - N² - Dic. 2007

Cuadro Nº1: Alumnos AC: Facilidades para el uso de las TICs

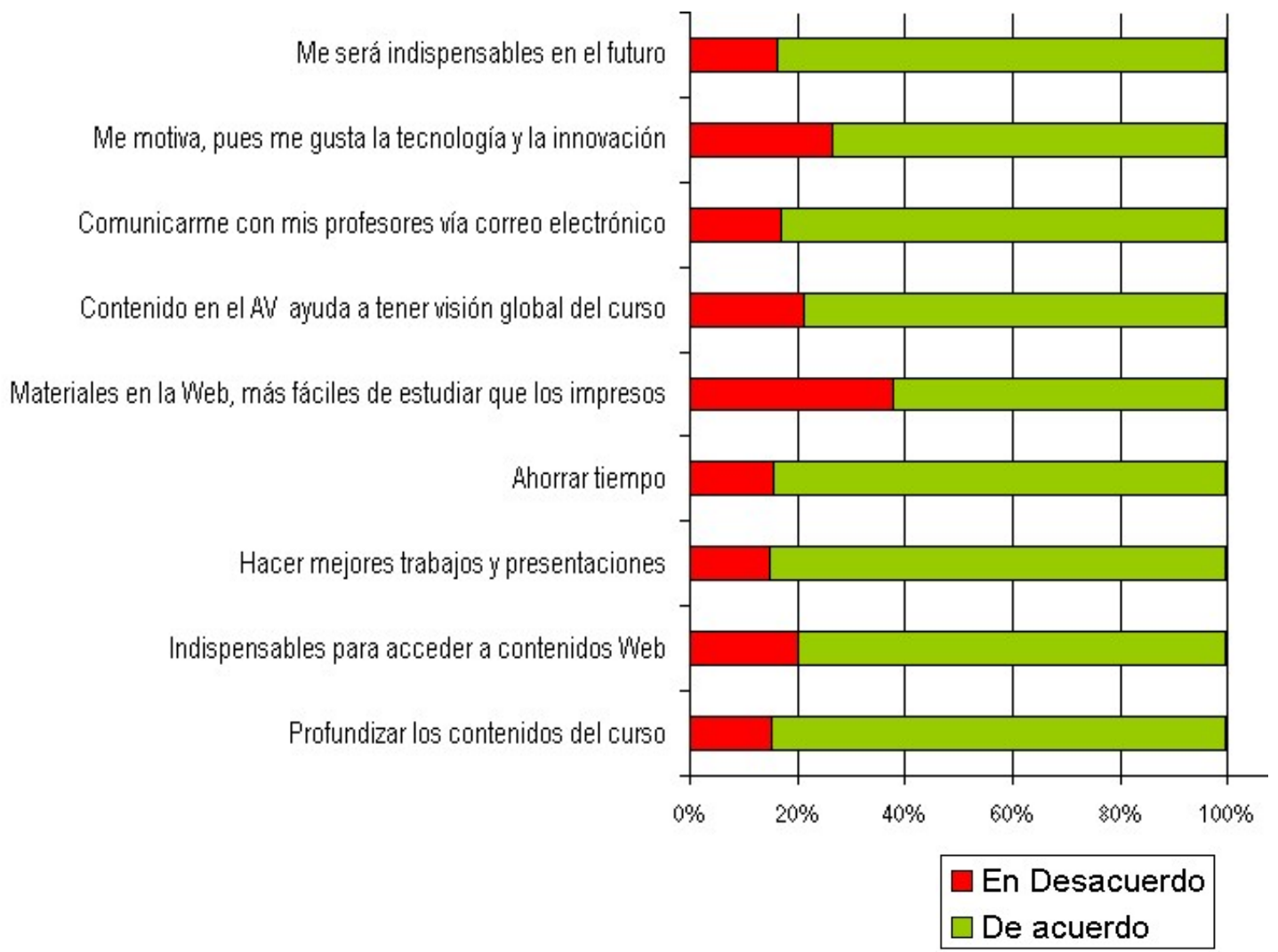

Fuente: Encuesta TICE, UPC (Diciembre, 2006)

Por su parte, los alumnos de EPE manifestaron, en promedio, un mayor grado de acuerdo ( $>4$ ) que los alumnos de Pregrado sobre las facilidades brindadas por las tecnologías para los estudios.

La proposición que mayor grado de acuerdo tuvo fue "Las TICs me serán indispensables en el futuro" (87,15\%), seguida por "Las TICs me permiten profundizar el contenido del curso" (87,05\%). Cabe resaltar que la proposición "Los materiales en el AV ayudan a tener una visión global del curso” recibió la mayor valoración (90\%) por parte de las mujeres de EPE, siendo, en líneas generales, la de mayor valoración recibida.

La proposición con menor grado de acuerdo en EPE, así como en AC, fue "Los materiales en la Web son más fáciles de estudiar que los materiales impresos” (32.91\%). 
Аก̃̃ 3 - №2- Dic. 2007

Cuadro N²: Alumnos EPE: Facilidades para el uso de las TICs

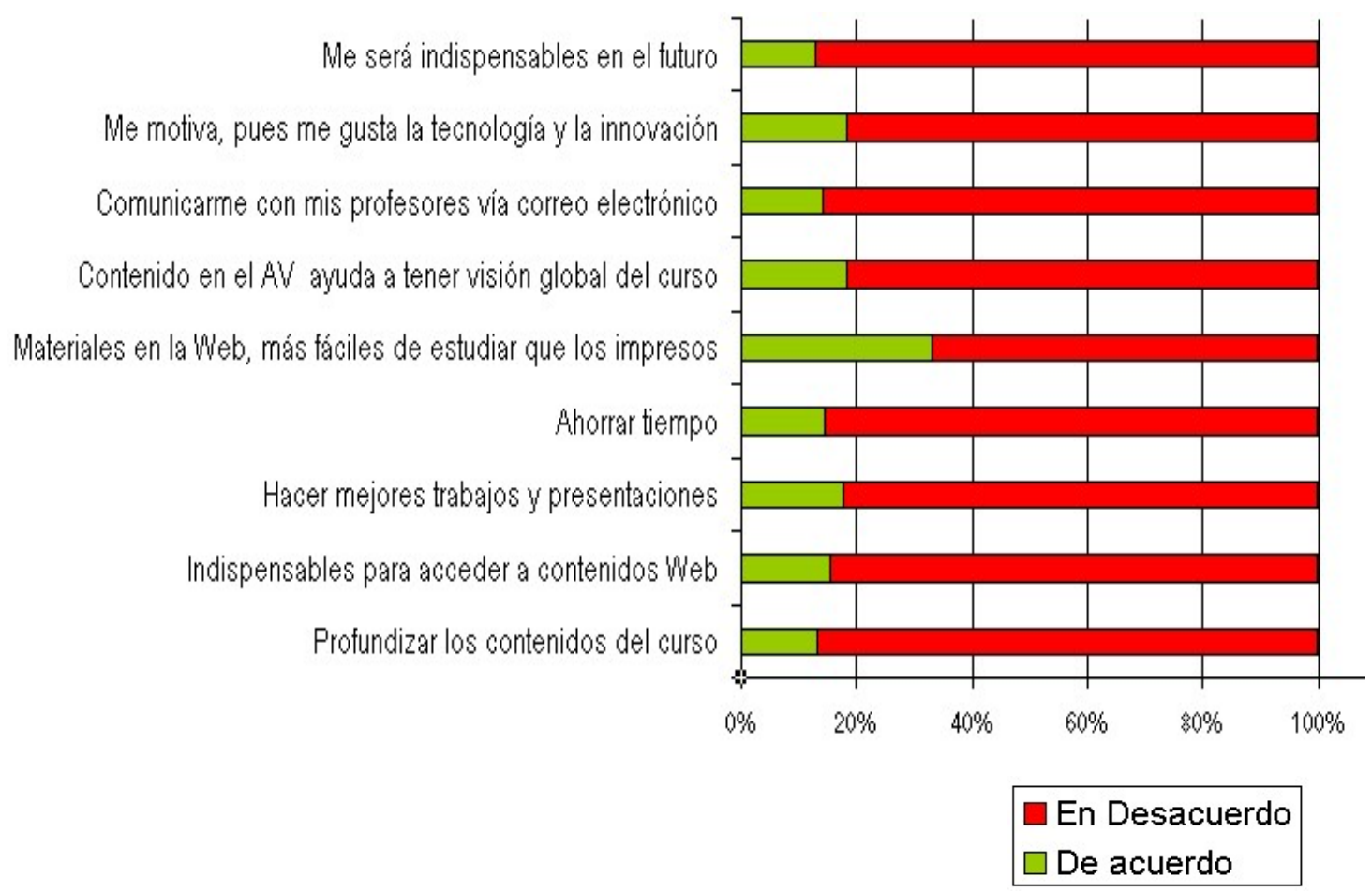

Fuente: Encuesta TICE, UPC (Diciembre, 2006)

En términos generales, las TICs fueron consideradas como herramientas que facilitaban el aprendizaje. Para los alumnos de AC, por su valor instrumental en la producción de trabajos; para los de EPE, como herramienta profesional. Un aspecto resaltable fue la valoración del uso de TICs para profundizar en los contenidos del curso y para contar con una visión global del mismo (dado que en el AV se cuenta con los materiales organizados). El punto que mayor discusión generó fue si los materiales en línea resultaban más fáciles de estudiar. Analizaremos ese tema a continuación.

\subsection{Obstáculos para el uso de TICs en la Educación}

En promedio, los alumnos encuestados expresaron su conformidad con las proposiciones referidas como obstáculos para el uso de TICs en la educación (AC=60,8\%, EPE=66.6\%). Dicho de otro modo, dichas proposiciones no fueron consideradas como obstáculos para el empleo de las TICs en sus estudios.

En el caso del Pregrado (AC), cabe destacar tres proposiciones que recibieron el mayor grado de acuerdo: "Los equipos y medios insuficientes para los estudiantes" (61\%), 
“Falta personal especializado para apoyar a los estudiantes” (54,8\%), y, finalmente, “Los materiales en la Web no reemplazan a los materiales impresos” (49,7\%).

Se destacaron también las respuestas de las mujeres del Pregrado, que manifestaron sentirse más “Cómodas con el uso de las TICs” (>6.8\%) que la población masculina. Asimismo, frente a la proposición "Las TICs no aportan nada a la calidad del proceso de enseñanza-aprendizaje”, la población femenina expresó un mayor desacuerdo (>7\%) que los varones. Finalmente, para las mujeres "La falta de apoyo especializado para los estudiantes” fue una proposición que obtuvo mayor porcentaje de acuerdo (>7\%) que en los hombres.

Cuadro No3: Alumnos AC: Obstáculos para el uso de las TICs

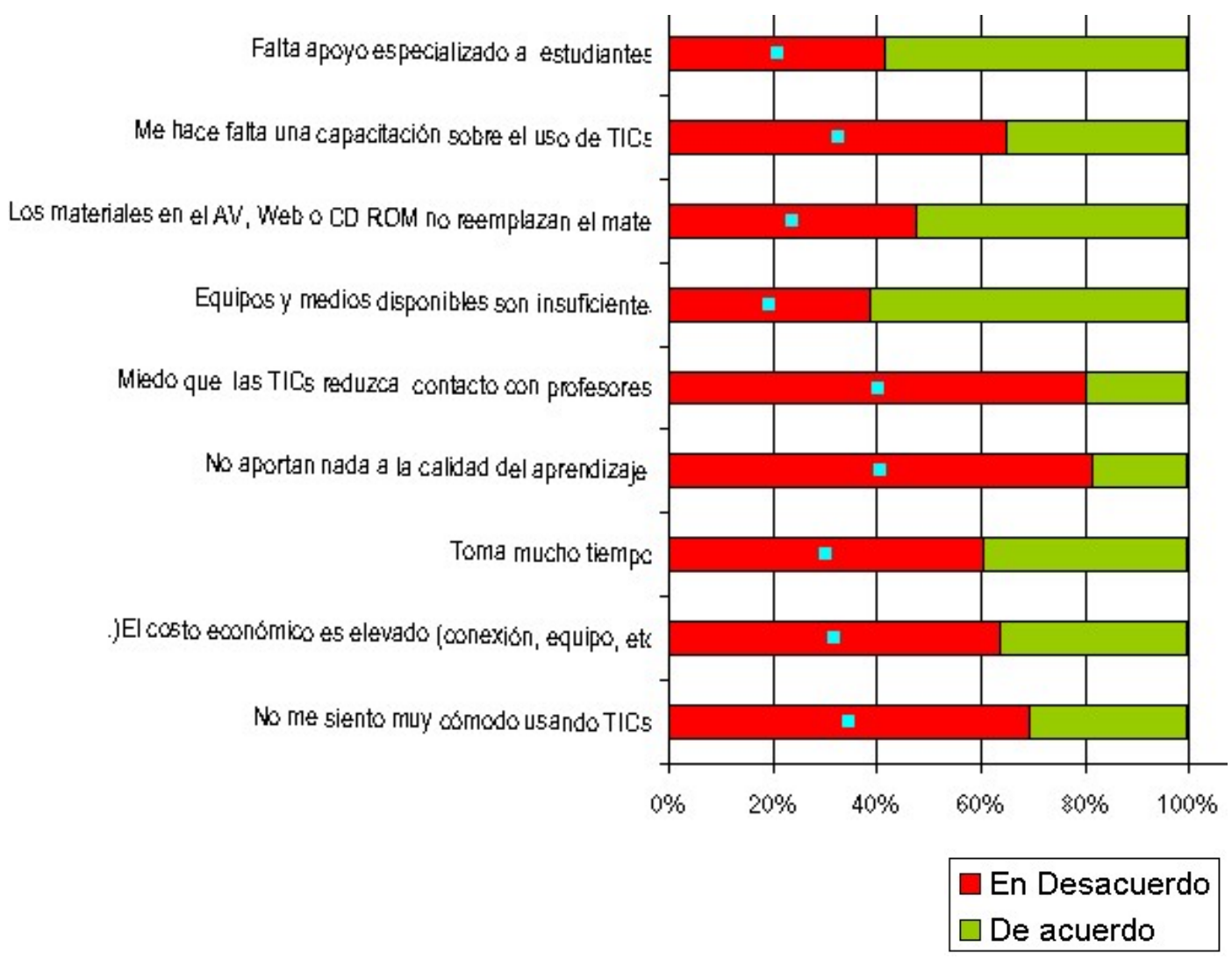

Fuente: Encuesta TICE, UPC (Diciembre, 2006)

Por su parte, los alumnos de EPE manifestaron un menor grado de acuerdo ( $>5 \%$ ) que los alumnos de AC respecto a las proposiciones presentadas como obstáculos para el uso de tecnologías en los estudios.

Sin embargo, las proposiciones que mayor acuerdo recibieron fueron "Los equipos y medios son insuficientes para los estudiantes" (49\%), "Hace falta personal 
especializado que apoye a los estudiantes (52,8\%), y, finalmente, "Los materiales en la Web no reemplazan a los materiales impresos" (52\%).

A nivel de género, los varones estuvieron de acuerdo en ( $>6 \%)$ que el "Costo económico de los equipo es elevado" era un obstáculo. Por su parte, las mujeres manifestaron un mayor desacuerdo ( $>7 \%$ ) frente a la proposición "Tengo miedo que las TICs reduzcan el contacto directo con mis profesores”. Cabe resaltar que dicha proposición fue la que alcanzó un mayor grado de desacuerdo (91\%).

Cuadro Nº: Alumnos EPE: Obstáculos para el uso de TICs

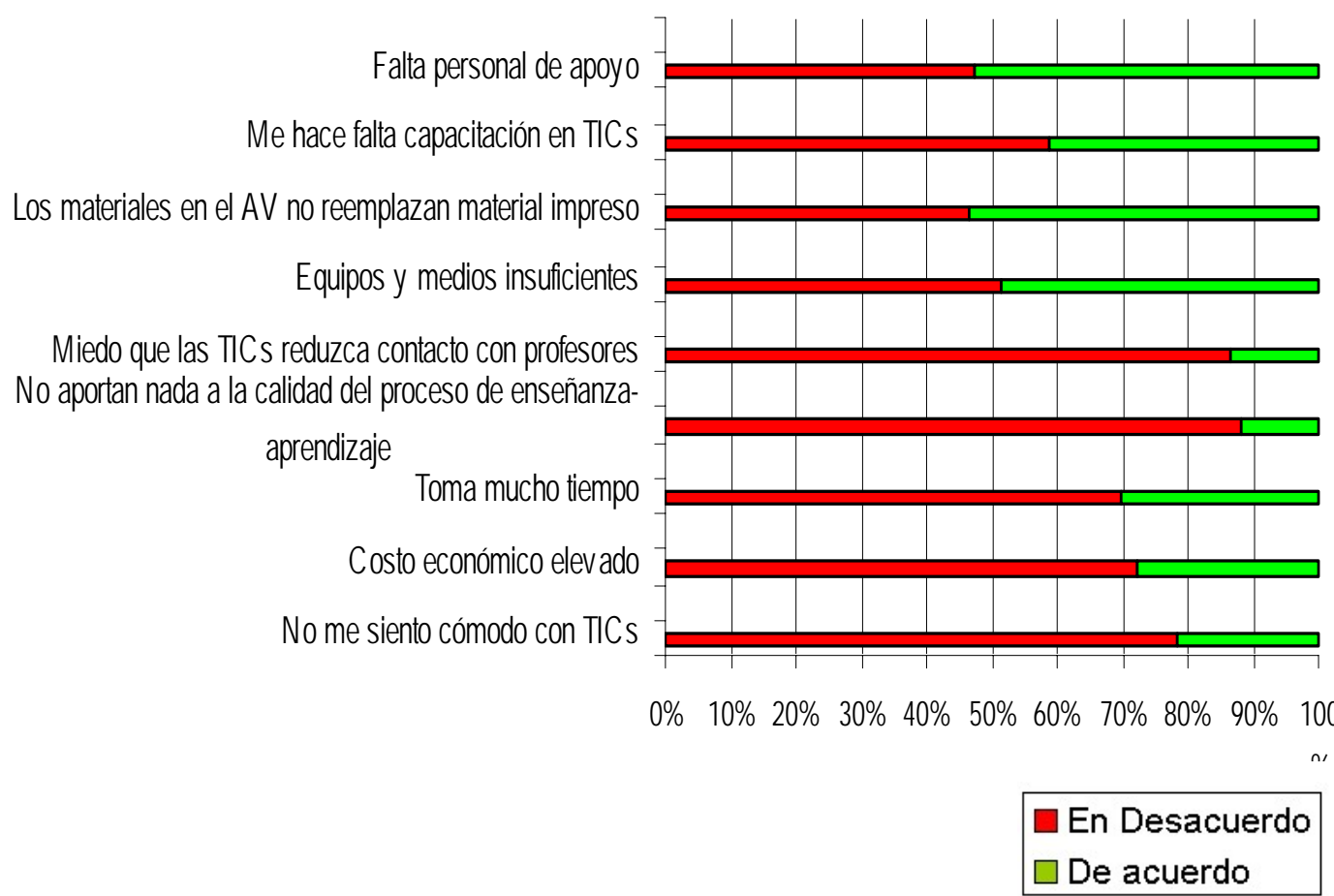

Fuente: Encuesta TICE, UPC (Diciembre, 2006)

En términos globales, los alumnos no consideraron que las proposiciones formuladas constituyeran obstáculos para el uso de TICs. No obstante, el tema de recursos, tanto físicos como humanos, fue valorado como un obstáculo, aunque cabe resaltar que representaban dificultades externas a los alumnos.

En este punto debe retomarse la reflexión de los materiales en línea como recursos que no reemplazan el material impreso. En un contexto donde la virtualización de los contenidos se impone, la tendencia nos empuja a colgar cada vez mayor material de estudio en el AV. La ventaja de esta práctica reside, más que en las actividades 
realizadas por el alumno en el AV, en la distribución del contenido y la accesibilidad del mismo. Estas consideraciones nos permiten avanzar hacia una apuesta de complementariedad, en la cual conviene conservar cierto tipo de materiales digitalizados en el $\mathrm{AV}$, pero también mantener los impresos que requieran ser manipulados físicamente por el alumno para apoyar su aprendizaje. La reflexión queda abierta.

\section{Empleo del Aula Virtual}

Un aspecto importante para comprender las prácticas respecto al empleo del AV en alumnos es el conocimiento de las condiciones de uso de esta herramienta. El resultado del estudio mostró una situación bastante favorable para los alumnos, pues, en promedio, el 95\% de los encuestados poseía computadora propia (75\% PC y 25\% laptop), siendo el lugar principal de conexión al AV su casa (71\%), seguido por el campus universitario (18\%).

\subsection{Propósito de empleo del AV}

Uno de los indicadores que el área de TICE monitorea de forma permanente es el tipo de empleo del AV. Este indicador es medido en términos cuantitativos, por las visitas registradas por tipo de herramienta en el AV. Luego, estas herramientas son agrupadas por su funcionalidad, obteniendo así tres grandes propósitos de uso:

- $\quad$ Contenidos: distribución, organización y descarga de materiales de estudio.

- $\quad$ Comunicación: interacción entre alumnos y docentes (correo, foro, chat).

- $\quad$ Actividades: envío de tareas, actividades en línea, evaluaciones, etc.

Los cuadros mostrados a continuación exhiben el historial de la distribución de estos tres propósitos de empleo. El cuadro 5 refleja la evolución del número de visitas por tipo de herramienta, mientras que el cuadro 6 nos permite comparar la proporción de cada propósito por ciclo. 
Cuadro No 5: Pregrado: Visitas por propósito de empleo del AV (2004-2006)

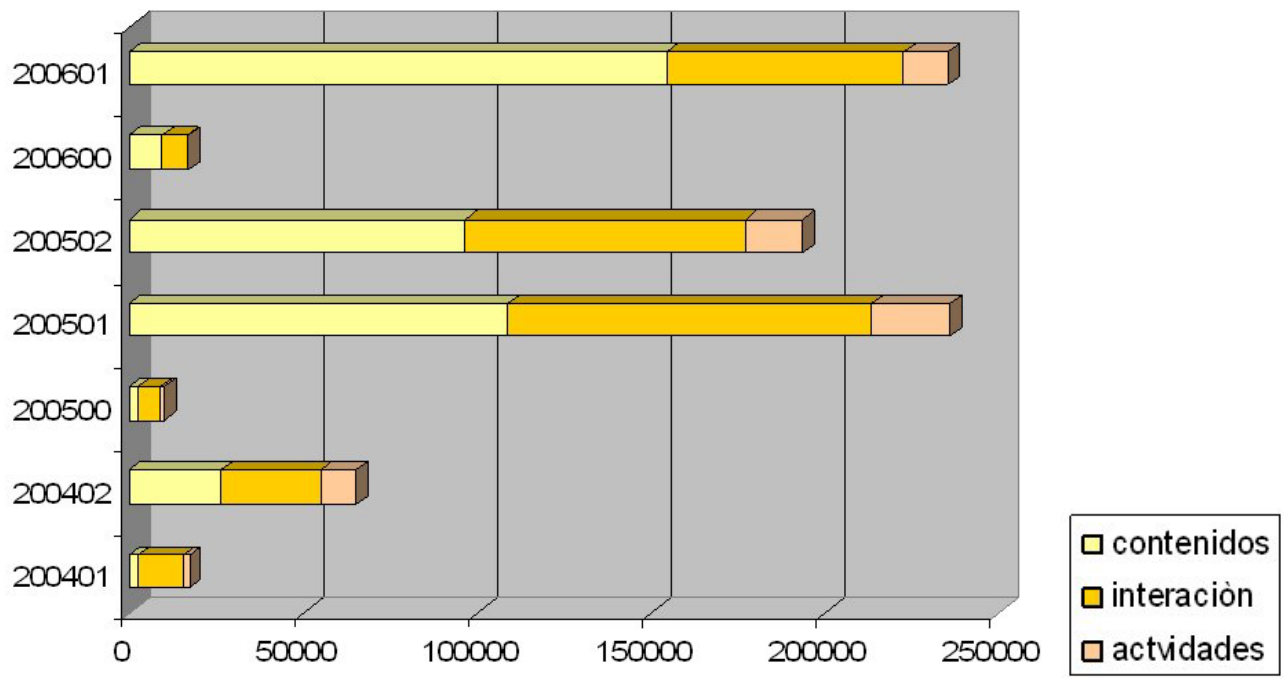

Fuente: Informe TICE, UPC (Marzo, 2007)

Cuadro No 6: Pregrado: Proporción de visitas según empleo del AV (2004-2006)

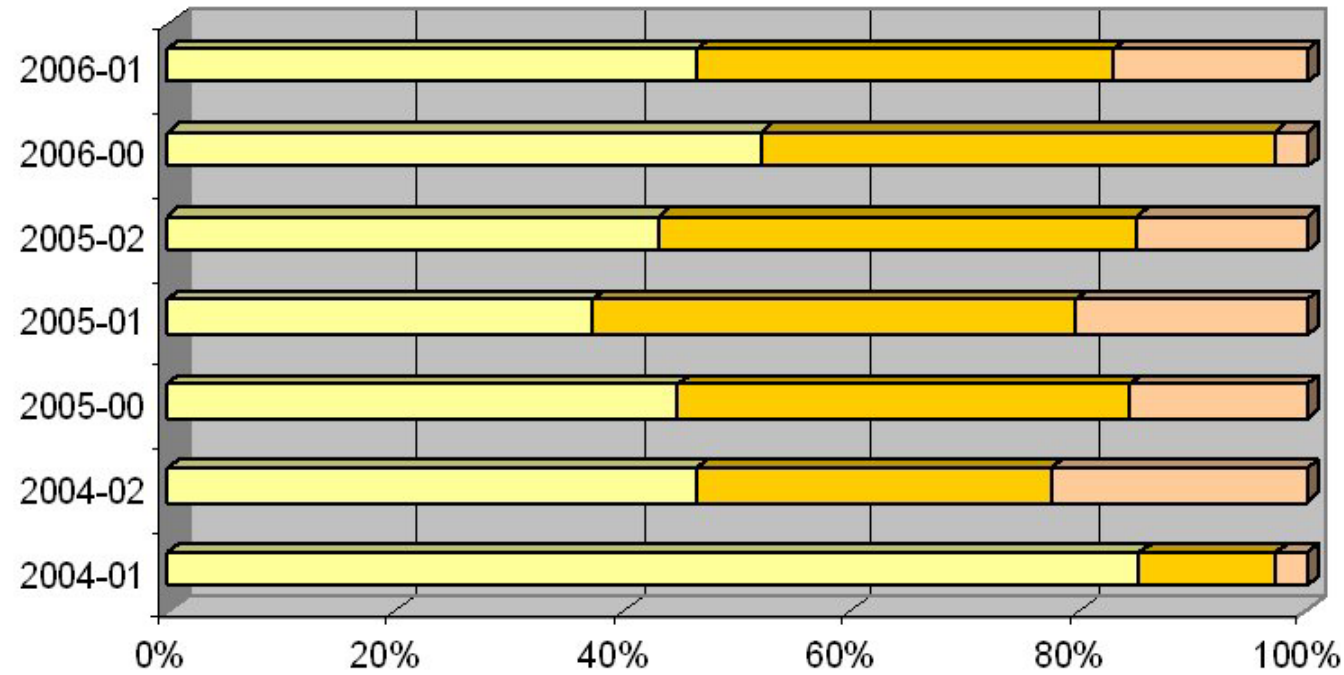

Fuente: Informe TICE, UPC (Marzo, 2007)

Siguiendo la propuesta de Lebrun ${ }^{11}$ sobre la vinculación entre los propósitos de empleo de la herramienta con el tipo de práctica pedagógica, se observa lo siguiente:

${ }^{11}$ Cfr. Lebrun 2006: 54 


\begin{tabular}{|l|l|}
\hline Propósito de uso & Práctica pedagógica \\
\hline Distribución de contenidos & Transmisión \\
\hline Comunicación & Interacción \\
\hline Actividades & Producción de saberes, pedagogía colaborativa \\
\hline
\end{tabular}

Para complementar esta observación cuantitativa, en el marco del estudio (IPM-TICE, 2006) se preguntó a los alumnos y docentes del Pregrado su propósito de uso del AV, obteniendo los siguientes resultados:

Tabla N²: Alumnos y Docentes AC: Propósito de empleo del AV

\begin{tabular}{|l|c|c|c|c|}
\hline \multirow{2}{*}{ Pregrado (AC) } & Contenidos & Comunicación & Actividades & Total \\
\hline Alumnos & $48,3 \%$ & $29,5 \%$ & $22,65 \%$ & $100 \%$ \\
\hline Docentes & $66,12 \%$ & $17,68 \%$ & $16,15 \%$ & $100 \%$ \\
\hline Promedio & $57,21 \%$ & $23,59 \%$ & $19,5 \%$ & $100 \%$ \\
\hline
\end{tabular}

Fuente: Encuesta TICE, UPC (Diciembre, 2006)

Cuadro No 7: Alumnos y Docentes AC: Propósito de empleo del AV

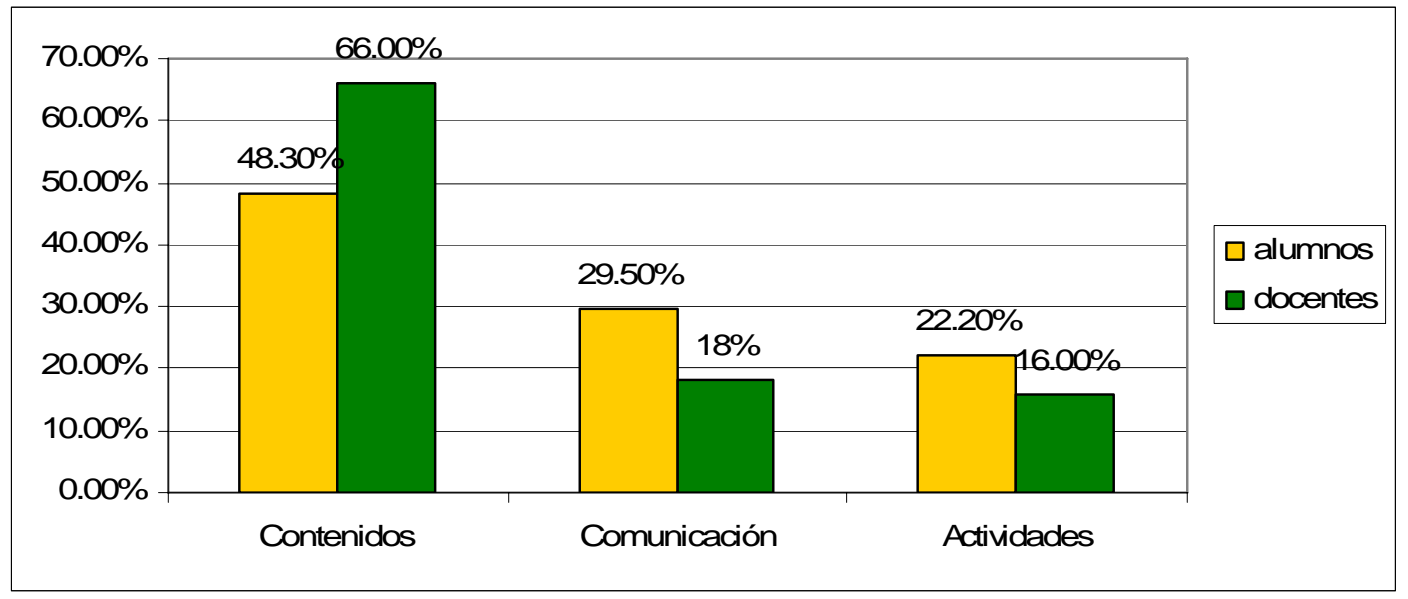

Fuente: Encuesta TICE, UPC (Diciembre, 2006)

De modo general, los propósitos de empleo se mantuvieron en una distribución similar a la observada en las visitas por herramientas. Sin embargo, resultó interesante 
Aña 3 - Nơ- Dic. 2007

comprobar las diferencias de uso del AV entre alumnos y docentes para la comunicación y la realización de actividades. Los alumnos expresaron un empleo más diverso de las herramientas que los profesores. Estos últimos se encontraban más concentrados en la provisión de contenidos del curso.

Ahora bien, para comprender mejor esta distribución, resulta importante reconocer que, durante una primera fase del ciclo académico, los docentes suelen dedicarse a alimentar el AV con contenidos. Solo posteriormente instalan una dinámica distinta con los estudiantes, en la cual descubren que pueden usar el AV para otros propósitos que el mero acceso a contenidos, tal como lo reflejó el testimonio del siguiente alumno:

“El Aula Virtual es un instrumento indispensable en el curso (...), ahí es donde la profesora (...) nos entregaba las diapositivas que usábamos cada clase, lo cual era de gran ayuda cuando teníamos una practica. También la profesora colgaba las asignaciones semanales. Aunque a muchos no les gustaba, estas asignaciones me ayudaban a entender las clases, puesto que debía practicar lo enseñado. Y últimamente me ha servido el foro del Aula Virtual para despejar todas mis dudas en el trabajo final, ya que mi profesora leía diariamente cualquier duda y las respondía rápidamente”. Delegado del Curso SI118, Relato de Experiencia 2006-1.

\subsection{Motivaciones para usar el AV}

Adicionalmente, se propuso a los docentes valorar 15 motivos que los impulsarían a disponer del AV en sus cursos. Entre ellas se encontraban tanto motivaciones internas como externas a los profesores. En ambos grupos de docentes, las proposiciones que mayor grado de acuerdo alcanzaron estuvieron vinculadas con motivaciones personales, como "La intención de mejorar el proceso de enseñanza-aprendizaje” y "El interés por usar una pedagogía más activa". Vale la pena resaltar que las motivaciones vinculadas directamente con la práctica pedagógica (realizar innovaciones, mejorar el proceso, variar el curso, etc.), fueron más fuertes en el caso de los docentes de AC que en los de EPE.

Las proposiciones externas, provenientes de sus pares o de los propios alumnos, tuvieron, en general, un menor grado de acuerdo. Sin embargo, para los docentes de EPE el motivo "Los estudiantes me han solicitado usar el Aula Virtual” alcanzó un grado de acuerdo de $37,50 \%$ frente a un $28 \%$ en AC. 
Аก̃ם 3 - N² - Dic. 2007

En líneas generales, tomando en cuenta las motivaciones resaltadas por los docentes, el uso del AV fue percibido como instrumento de mejora e innovación en la actividad pedagógica.

\section{Valor pedagógico del AV}

La mayor parte del estudio (IPM-TICE, 2006) se concentró en el planteamiento de 32 proposiciones a los usuarios. Estas estuvieron referidas a los seis tipos de finalidades de uso del AV (motivación, organización, contenidos, construcción, comunicación y seguimiento), ante las cuales debían expresar su grado de acuerdo o desacuerdo. La valoración de los usuarios sobre estas proporciones permitió reconocer la percepción sobre la utilidad pedagógica de esta herramienta en el proceso de enseñanzaaprendizaje.

En el cuadro siguiente se presentan las proposiciones más valoradas por los alumnos. Se observa que la valoración fue homogénea en ambos grupos de alumnos, pues no se presentaron diferencias sustanciales en sus respuestas. En ambos casos, la proposición más valorada fue "Puedo seguir mejor la secuencia del curso", lo que indica una percepción de utilidad relacionada con el apoyo a la organización del aprendizaje. Por otro lado, existió consenso respecto a las finalidades de uso más valoradas, como la provisión de contenidos y motivación. A esta le siguieron otras, como la organización y construcción del conocimiento. Cabe resaltar que la finalidad del seguimiento no se encontró necesariamente reflejada en las proposiciones más valoradas.

Tabla N³: Alumnos AC -EPE

Proposiciones con mayor valor pedagógico (de acuerdo)

\begin{tabular}{|l|c|c|c|c|}
\hline Proposiciones & AC & EPE & Diferencias & Finalidad \\
\hline $\begin{array}{l}\text { Puedo seguir mejor la secuencia del } \\
\text { curso. }\end{array}$ & $78.2 \%$ & $66.4 \%$ & $11.7 \%$ & Organización \\
\hline $\begin{array}{l}\text { Puedo repasar mejor las ideas } \\
\text { trabajadas en clase. }\end{array}$ & $72.9 \%$ & $66.3 \%$ & $6.6 \%$ & Contenidos \\
\hline $\begin{array}{l}\text { Las informaciones propuestas son más } \\
\text { concretas (relacionadas con la vida } \\
\text { cotidiana, la vida profesional, etc.). }\end{array}$ & $71.1 \%$ & $64.7 \%$ & $6.4 \%$ & Motivación \\
\hline $\begin{array}{l}\text { Encuentro los contenidos del curso de } \\
\text { manera organizada. }\end{array}$ & $66.6 \%$ & $62.0 \%$ & $4.6 \%$ & Contenidos \\
\hline Hay más trabajos grupales que & $65.6 \%$ & $61.7 \%$ & $3.9 \%$ & Construcción \\
\hline
\end{tabular}




\begin{tabular}{|l|c|c|c|c|}
\hline realizar. & & & & \\
\hline Me siento más motivado. & $65.3 \%$ & $61.5 \%$ & $3.8 \%$ & Motivación \\
\hline El dictado de clases se reduce. & $65.2 \%$ & $57.4 \%$ & $7.7 \%$ & Organización \\
\hline $\begin{array}{l}\text { Las tareas que nos propone el profesor } \\
\text { son más interesantes. }\end{array}$ & $65.0 \%$ & $57.4 \%$ & $7.6 \%$ & Motivación \\
\hline $\begin{array}{l}\text { Aprendo a usar mejor las tecnologías. } \\
\begin{array}{l}\text { Los recursos que el profesor propone } \\
\text { son más variados. }\end{array}\end{array}$ & $62.1 \%$ & $55.7 \%$ & $6.4 \%$ & Construcción \\
\hline
\end{tabular}

Fuente: Encuesta TICE, UPC (Diciembre, 2006)

El testimonio que presentamos a continuación resumió, en cierto modo, la percepción de los estudiantes sobre el valor pedagógico del AV:

"El Aula Virtual me parece una muy buena idea, ya que es bastante útil para el envío del material que se utiliza en clase. A veces, por falta de tiempo, el correo electrónico se llena y no llega la información. Además, acá la información se encuentra distribuida por cursos, está más ordenada y facilita su búsqueda”. Alumna del Curso HU94, Relato de experiencia 20.

Ahora corresponde presentar las proposiciones menos valoradas por los estudiantes. Sobre este punto se pudo apreciar también una homogeneidad en la percepción de los estudiantes de AC y EPE. Asimismo, la finalidad de uso de la herramienta como apoyo a la comunicación y el seguimiento fueron los tipos de ayuda que menos porcentaje obtuvieron.

Tabla No 4

Alumnos AC -EPE: Proposiciones con menor valor pedagógico

\begin{tabular}{|lcccc|}
\hline Proposiciones & AC & EPE & Diferencias & Finalidad \\
\hline Tengo mayor autonomía para estudiar. & $49.3 \%$ & $46.1 \%$ & $3.2 \%$ & Organización \\
Más estudiantes participamos & & & & \\
activamente en el curso. & $48.9 \%$ & $46.1 \%$ & $2.8 \%$ & Comunicación \\
Soy más activo en el curso. & $47.6 \%$ & $46.0 \%$ & $1.6 \%$ & Motivación \\
Aprendo más. & $46.3 \%$ & $44.9 \%$ & $1.3 \%$ & Motivación \\
Mi aprendizaje es de mayor calidad. & $45.4 \%$ & $43.9 \%$ & $1.5 \%$ & Construcción \\
\hline
\end{tabular}


Añ̃ 3 - N02- Dic. 2007

Las evaluaciones se basan más en el

trabajo que presentamos los

estudiantes.

$44.1 \%$

$43.3 \%$

$0.7 \%$

Seguimiento

El profesor puede evaluar mejor mi

trabajo.

$37.0 \%$

$39.1 \%$

$-2.0 \%$

Seguimiento

Tengo mayor interacción con el

profesor.

$36.2 \%$

$34.3 \%$

$1.9 \%$

Comunicación

Las actividades son más

personalizadas.

$31.1 \% \quad 32.6 \%$

$-1.4 \%$

Motivación

Fuente: Encuesta TICE, UPC (Diciembre, 2006)

La percepción de los alumnos guardó relación con los propósitos de empleo del AV identificados en el punto anterior, confirmando una dinámica en la que el valor pedagógico se centraba en los contenidos y organización del estudio. La comunicación entre pares y docentes, así como el seguimiento de los alumnos, son dinámicas que, aparentemente, no están siendo apoyadas con el uso de esta herramienta.

A continuación, presentamos las proposiciones con mayor valoración por parte de los docentes. De modo general, pudo constatarse que, si bien la percepción de los profesores se mostró homogénea, existieron mayores diferencias entre los resultados de los docentes de AC y EPE que entre los alumnos de dichas modalidades de estudio. De otro lado, las proposiciones valoradas por los docentes no coincidieron con las elegidas por los alumnos; sin embargo, sí existió correspondencia respecto a las finalidades de uso. Para ambos grupos de docentes, la proposición con mayor valoración fue "Los estudiantes dedican más tiempo a realizar sus trabajos con profundidad”, la cual coincidió con el apoyo en la organización del estudio. La percepción de utilidad de la herramienta se refirió a las finalidades de provisión de contenidos, construcción del conocimiento y motivación. Al igual que en el caso de los alumnos, las finalidades de apoyo a la comunicación y seguimiento no destacaron entre las más valoradas. Vale la pena resaltar que los docentes percibieron un mayor grado de acuerdo respecto a la proposición “Tengo mayor interacción con mis alumnos”, pese a que la misma estuvo entre las menos ponderadas por los alumnos. Retomaremos el análisis de ese punto más adelante. 
Tabla No 5: Docentes AC -EPE

Proposiciones con mayor grado de acuerdo

\begin{tabular}{|l|c|c|c|c|}
\hline $\begin{array}{l}\text { Proposiciones } \\
\text { Los estudiantes dedican más tiempo } \\
\text { profundizar trabajos con mayor }\end{array}$ & $85.7 \%$ & $82.6 \%$ & $3.1 \%$ & Organización \\
\hline $\begin{array}{l}\text { Los estudiantes repasan las ideas } \\
\text { trabajadas en clase con ayuda de los } \\
\text { materiales disponibles. }\end{array}$ & $85.4 \%$ & $77.2 \%$ & $8.1 \%$ & Contenidos \\
\hline $\begin{array}{l}\text { Solicito a los estudiantes más } \\
\text { actividades de investigación. }\end{array}$ & $83.8 \%$ & $76.1 \%$ & $7.6 \%$ & Construcción \\
\hline $\begin{array}{l}\text { Propongo actividades más próximas } \\
\text { a la vida profesional. }\end{array}$ & $83.3 \%$ & $75.0 \%$ & $8.3 \%$ & Motivación \\
\hline $\begin{array}{l}\text { Tengo más interacción con mis } \\
\text { estudiantes. }\end{array}$ & $82.2 \%$ & $73.9 \%$ & $8.3 \%$ & Comunicación \\
\hline $\begin{array}{l}\text { Los estudiantes aprenden más. } \\
\text { Organizo más actividades de } \\
\text { aprendizaje cooperativo. }\end{array}$ & $80.95 \%$ & $70.00 \%$ & $10.95 \%$ & Construcción \\
\hline $\begin{array}{l}\text { Propongo a mis estudiantes tareas o } \\
\text { actividades más interesantes. }\end{array}$ & $77.50 \%$ & $70.00 \%$ & $7.50 \%$ & Motivación \\
\hline $\begin{array}{l}\text { Me ayuda a reducir la parte } \\
\text { expositiva de mis cursos. }\end{array}$ & $70.00 \%$ & $8.05 \%$ & Construcción \\
\hline $\begin{array}{l}\text { Propongo a los estudiantes recursos } \\
\text { más variados. }\end{array}$ & $70.00 \%$ & $5.76 \%$ & Organización \\
\hline
\end{tabular}

Fuente: Encuesta TICE, UPC (Diciembre, 2006)

En cuanto a las proposiciones menos valoradas por los docentes, se mantuvo una diferencia mayor entre la percepción de los docentes de AC y EPE que en el caso de los alumnos. Otro aspecto resaltable fue el hecho que más del 50\% de los profesores de AC manifestaran su acuerdo sobre el conjunto de las proposiciones. En el caso de EPE, los docentes expresaron un grado de de acuerdo considerablemente menor que los docentes del Pregrado, siendo las siguientes las proposiciones con menos ponderación respecto a su utilidad pedagógica: "Los estudiantes desarrollan más competencias para la 
búsqueda de información" (35\%) y "Hay una mayor participación activa en el curso" (35\%). Por último, las finalidades de uso asociadas a las respuestas de los alumnos en este caso fueron, en primer lugar, la construcción del conocimiento, seguido de la motivación, el seguimiento, la organización y la comunicación.

Tabla No 6: Docentes AC -EPE

Proposiciones con menor valoración de acuerdo

\begin{tabular}{|l|c|c|c|c|}
\hline Proposiciones & AC & EPE & Diferencia & Finalidad \\
\hline $\begin{array}{l}\text { Propongo informaciones más } \\
\text { concretas en relación a la vida } \\
\text { cotidiana y profesional. }\end{array}$ & $58.5 \%$ & $45.0 \%$ & $13.5 \%$ & Motivación \\
\hline $\begin{array}{l}\text { Mis estudiantes están más } \\
\text { motivados. }\end{array}$ & $57.5 \%$ & $45.0 \%$ & $12.5 \%$ & Motivación \\
\hline $\begin{array}{l}\text { Los estudiantes están más } \\
\text { entrenados para el trabajo en } \\
\text { equipo. }\end{array}$ & $57.5 \%$ & $45.0 \%$ & $12.5 \%$ & Construcción \\
\hline $\begin{array}{l}\text { El aprendizaje es de mayor } \\
\text { calidad. }\end{array}$ & $55.0 \%$ & $40.0 \%$ & $15.0 \%$ & Construcción \\
\hline $\begin{array}{l}\text { Doy mejores y más frecuentes } \\
\text { asesorías a los alumnos. }\end{array}$ & $52.5 \%$ & $38.1 \%$ & $14.4 \%$ & Seguimiento \\
\hline $\begin{array}{l}\text { Los estudiantes siguen mejor la } \\
\text { secuencia del curso porque tienen } \\
\text { el sílabo y el cronograma } \\
\text { disponible. }\end{array}$ & $52.5 \%$ & $38.1 \%$ & $14.4 \%$ & Organización \\
\hline $\begin{array}{l}\text { Los estudiantes desarrollan más } \\
\text { competencias para la búsqueda de } \\
\text { información. }\end{array}$ & $35.0 \%$ & $17.3 \%$ & Construcción \\
\hline $\begin{array}{l}\text { Hay una mayor participación } \\
\text { activa en el curso. }\end{array}$ & & $35.0 \%$ & $16.2 \%$ & Comunicación \\
\hline Fuente: Encuesta TICE, UPC (Diciembre & & & & \\
\hline
\end{tabular}

Fuente: Encuesta TICE, UPC (Diciembre, 2006) 
Aก̃̃ 3 - N02- Dic. 2007

Finalmente, resultó interesante comparar las proposiciones que mayor diferencia obtuvieron en la apreciación entre los grupos encuestados. En la tabla siguiente, destacamos cuatro casos relevantes:

\section{Tabla No 7: Alumnos y docentes (AC/EPE)}

\section{Proposiciones con valoración diferente}

$\begin{array}{llll}\text { Proposiciones / finalidad } & \text { Población } & \text { Pregrado } & \text { EPE } \\ \text { Autonomía para estudiar } & \text { Alumnos } & 49.3 \% & 46.1 \% \\ \text { (Organización). } & \text { Docentes } & 70.7 \% & 55 \% \\ \text { Seguir mejor la secuencia del curso en } & \text { Alumnos } & 75.2 \% & 66.4 \% \\ \text { el AV (Organización). } & \text { Docentes } & 52.5 \% & 60 \% \\ \text { Hay mayor interacción entre alumnos y } & \text { Alumnos } & 36.2 \% & 34.3 \% \\ \text { docentes (Comunicación). }{ }^{12} & \text { Docentes } & 82.2 \% & 82.6 \% \\ \text { Las evaluaciones se basan más en el } & \text { Alumnos } & 37.0 \% & 39.1 \% \\ \text { trabajo que presentamos los estudiantes } & & & 70 \% \\ \text { (Seguimiento). } & \text { Docentes } & 65.1 \% & \end{array}$

Fuente: Encuesta TICE, UPC (Diciembre, 2006)

- Autonomía para estudiar: Mientras los docentes de AC percibieron que el AV permitía a los alumnos tener mayor autonomía para el estudio (70\%), los alumnos de AC no estuvieron de acuerdo respecto a esta utilidad (49\%). En EPE la tendencia fue la misma, pero la diferencia entre la percepción de alumnos y docentes es menos importante que en el caso del pregrado. Esta constatación nos hizo reflexionar sobre la preparación que tienen, en general, los jóvenes alumnos de AC (entre 16 y 21 años) para ejercer con autonomía su proceso de enseñanzaaprendizaje. Si bien el AV provee recursos para la organización y planificación de tiempos y materiales, ello no implica que los alumnos lo empleen para adquirir mayor autonomía en sus estudios.

- Seguir mejor la secuencia del curso: En este caso se produjo lo contrario. Los alumnos de AC manifestaron estar de acuerdo con esta proposición en un 75\%, mientras los docentes de AC solo le otorgaron un 52\%. En EPE la tendencia fue la misma, aunque en este caso también resalta que las diferencias entre las percepciones de alumnos y docentes son menores a las encontradas en el caso del

\footnotetext{
${ }^{12}$ Cabe resaltar que este fue el ítem con mayor grado de acuerdo entre los docentes.
} 
pregrado. Estos resultados nos invitan a indagar sobre la funcionalidad percibida por cada grupo, así como sobre la dinámica que implica tener los contenidos organizados en el AV. Mientras los alumnos valoraron tener todos los materiales organizados desde un principio del curso para seguir mejor su secuencia y tener una visión global del mismo (ver resultados del cuadro 2), los docentes, en la práctica, no organizan sus contenidos con anticipación, “colgando” los documentos conforme avanza el ciclo.

- Interacción con los profesores: En este punto se encontraron las diferencias más saltantes. Mientras la mayoría de docentes de AC y EPE (80\%) señalaron que con el AV tenían mayor interacción con sus alumnos, solo la tercera parte de estudiantes de dichas modalidades estuvieron de acuerdo con ellos.

Por otro lado, los profesores confirmaron, a través de los focus group y en los relatos de experiencias, la importancia que encontraban en el uso del AV como herramienta de comunicación. Así se mostró en los siguientes testimonios:

"La interacción entre los alumnos y el profesor ha sido potenciada por el Aula Virtual, brindando más canales para dicha interacción”. (Profesor del curso Modelo Educativo, Relato de Experiencia 2004-2).

"Este medio nos permitió ampliar nuestro espacio de interacción de manera muy importante, sin afectar el espacio correspondiente a las sesiones presénciales“. (Profesor del curso Estadística Aplicada a los Negocios, Relato de Experiencia 2005-2).

"Los medios virtuales se presentan como aquella herramienta que complementa el trabajo que realizamos en las sesiones de clase, ya que muchas veces, por tiempo o temores respecto a la participación de los alumnos, quedan temas sin profundizar”. Profesor del curso HU94, Relato de experiencia 2006-1.

El AV fue percibido como un punto de encuentro en el cual era posible prolongar el espacio de comunicación en clase. Sin embargo, habría que profundizar más en el tipo de comunicación que se realiza “virtualmente" para comprender la diferencia de percepción entre alumnos y docentes sobre este punto. Por el lado de los alumnos, una explicación posible estaría en la expectativa de contar con una interacción más personalizada, como la que se practica en el Chat u otros medios virtuales. En el caso de los profesores, estos suelen utilizar dichos medios para enviar información de forma masiva (para todo el grupo). Sin duda, la interacción en los medios virtuales es un campo de investigación emergente que aportará 
pistas para diseñar dinámicas de comunicación que apoyen el proceso de enseñanza-aprendizaje.

- Las evaluaciones se basan más en los trabajos que presentan los estudiantes: En este aspecto también se revelaron diferencias importantes en las que coincidían los alumnos y docentes de ambos grupos. Mientras el 66\% de docentes de AC y EPE consideró que con el AV sus evaluaciones se basaban más en el trabajo de los estudiantes, solo la tercera parte de los alumnos de las mismas modalidades estuvieron de acuerdo con dicha proposición. Si bien el tema de la evaluación ha sido tradicionalmente un punto critico en la cultura del aprendizaje, es conveniente preguntarse si los docentes solicitan a los alumnos publicar sus trabajos en el AV, y si se establecen criterios claros para que estos puedan reconocer cómo están siendo evaluados.

\subsection{Comentarios de los alumnos del Pregrado regular (AC)}

Por último, el estudio incluyó una opción abierta para comentarios o sugerencias. Luego de analizar los contenidos, se obtuvieron 33 categorías de respuestas que, para fines de esta investigación, agrupamos en dos categorías principales: aspectos técnicos y aspectos culturales (contenidos, actividades, difusión, capacitación). Finalmente, se incluyó una categoría denominada “Otros”. Los resultados se distribuyeron de la siguiente manera: 50\% para los aspectos técnicos, 43\% para los culturales, y 7\% para los otros.

Dentro de la categoría “aspectos técnicos”, el ítem que más comentarios tuvo fue la lentitud del AV, mencionada en un 19,6\% por los alumnos y en un 15\% por los docentes. Por su parte, en “aspectos culturales”, la mayoría de los alumnos refirió que los profesores no empleaban el Aula Virtual, lo cual se correspondió con las sugerencias más repetidas por los docentes respecto a la necesidad de una mayor capacitación para el uso de la herramienta. El siguiente comentario ilustró bien estos resultados: "El Aula Virtual es una herramienta valiosa, interesante y que será de mucha utilidad para su proceso de aprendizaje, siempre y cuando la usen todos los profesores" (Focus group: Percepción de los alumnos sobre las Aulas Virtuales, Área de Investigación y Evaluación, DECE. Julio de 2004).

De este modo, los comentarios permitieron evidenciar dos factores críticos e indispensables para la creación de condiciones apropiadas para el uso del AV.: un adecuado soporte técnico y una permanente capacitación para los profesores. 


\section{Discusión final}

Los resultados obtenidos a través de las diferentes fuentes empleadas, nos permiten constatar que el Aula Virtual es percibida por alumnos y docentes como una herramienta útil. El crecimiento cuantitativo de su empleo en la UPC, la percepción de las TICs como herramienta de apoyo en la educación, la identificación de sus propósitos de uso y la percepción de su valor pedagógico han dejado testimonio de que el AV ha logrado tener una presencia positiva en su proceso de enseñanza-aprendizaje.

Por otro lado, resulta interesante destacar las diferencias encontradas entre las percepciones de de modalidades encuestadas (Pregrado y EPE); por ejemplo, los

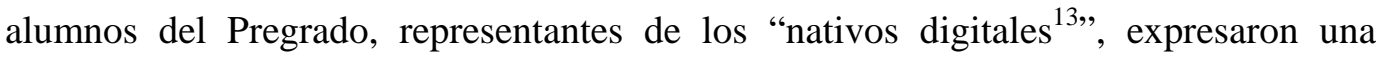
opinión más favorable respecto a la utilidad pedagógica del AV, mientras los alumnos de EPE, en su mayoría adultos pertenecientes a la categoría de "inmigrantes digitales", mostraron un grado de acuerdo más bajo. Por ello, es importante resaltar que la noción de "valor pedagógico" se construye a partir de una experiencia concreta del uso de la herramienta en una situación de aprendizaje, lo cual nos invita a plantear estrategias diferenciadas para cada grupo de usuarios.

Respecto a los tipos de ayuda más empleados, ha podido destacarse que el AV suele ser utilizada, principalmente, como una herramienta que facilita la organización y provisión de contenidos. En segundo lugar, se le ha resaltado como un factor de motivación y facilitación de las actividades de aprendizaje (construcción del conocimiento). Finalmente, ha quedado también demostrado que no es aún lo suficientemente explotada como herramienta que facilita la comunicación (en términos de interacción) y el seguimiento del aprendizaje. Esto nos ha llevado a reflexionar, por un lado, si la forma de uso del AV refleja algún tipo de práctica privilegiada y, por otro, si la falta de capacitación y de tiempo para utilizarla explica un empleo limitado de la herramienta. En ambos casos, se ha podido concluir que el AV es una herramienta que ayuda a amplificar la práctica pedagógica, pero que los grandes beneficios generados por su sola presencia no hacen la diferencia en la evolución de estas prácticas. Sin lugar a dudas,

\footnotetext{
${ }^{13}$ El termino "Nativos Digitales" hace referencia a los que nacieron en un mundo con computadoras e interactúan con las TICs e Internet y los videojuegos. El resto, los que no nacimos en el mundo digital pero tenemos de cierta manera integradas en nuestras vidas el uso de la nueva tecnología, somos Inmigrantes Digitales. La génesis de estos conceptos acuñados por Mark Prensky, se remonta a los años1994-96 y en los últimos años han sido desarrollado en profundidad, reflejando con acierto hacia dónde están evolucionando las tecnologías de la información y la comunicación, y esbozando los rasgos de nuestros usuarios en un contexto digital.
} 
exige la renovación de las competencias de los usuarios sobre diversos planos: diseño instruccional, comunicación, elaboración de materiales digitales, entre otros.

Finalmente, consideramos que la evolución de la tecnología y las innovaciones en las prácticas pedagógicas requieren estar soportadas por una investigación y evaluación permanente. En ese sentido, este trabajo constituye un punto de referencia inicial para futuros estudios sobre la integración del Aula Virtual en la educación superior.

Agradecimientos:

A Enith Huamán, Jefe del Área de Investigación del Vicerrectorado Académico. A Melissa Santos y Miguel Ángel Estacio, por su apoyo en el tratamiento estadístico de los resultados.

\section{Bibliografía}

BATES, A. W.

2002 Cómo gestionar el cambio tecnológico: Estrategias para los responsables de centros universitarios, Ed. Gedisa, Barcelona, 2001.

BADIA, Antoni

2006 Ayudar a aprender con tecnología en la educación superior». En: Antoni BADIA (coord.). Enseñanza y aprendizaje con TIC en la educación superior [monográfico en línea]. Revista de Universidad y Sociedad del Conocimiento (RUSC). Vol. 3, n. ${ }^{\circ}$ 2. UOC. <http://www.uoc.edu/rusc/3/2/dt/esp/badia.pdf>

DOCQ, Francoise, MARCEL Lebrun y Denis SMIDS.

2007 Claroline, une plate-forme d'enseignement-apprentissage sur Internet. Pour propulser la pédagogie active et l'iinovation?. En: Actes du 4eme Colloque “Questions de pédagoges dans l’enseignement supérieur”, Enero 2007, Louvain la-Neuve.

\section{LEBRUN, M.}

2002 Théories et méthodes pédagogiques pour enseigner et apprendre. Quelles place pour les TICs dans l'éducation. Ed. De Boeck Université, Bruselas, 2002. 
Aña 3 - Nơ- Dic. 2007

2004 The Emerging Online Life of the Digital Native: What they do differently because of technology, and how they do it A work in progress. [Fecha de consulta: 21/6/07].

$<$ http://www.marcprensky.com/writing/PrenskyThe_Emerging_Online Life_of_the_Digital_Native-03.pdf $>$

RADA, R.

2001 Understanding Virtual Universities, Ed. Bristol: Intellect books.

TSCHANG, F. Ted et T. DELLA SENTA.

200| Access to Knowledge: New Information Technologies and the Emergence of the Virtual University. Ed. UNU/IAS, Holanda.

SULMONTt, L.

(2004): La Universidad en la Era Virtual y el desarrollo de dispositivos de formación.

En: Revista Iberoamericana de Educación a Distancia (RIED), N 1/2, Vol, 7.

Págs.163-173

TICE, Departamento de Calidad Educativa

2007 Informe TICEsobre el uso del Aula Virtual 2004-2006. Marzo 2007, UPC.

2006 Infome TICE sobre encuesta Aula Virtual, Diciembre 2006, UPC

Para citar este documento, puede utilizar la siguiente referencia:

SULMONT, Leao (2007). “El Valor pedagógico del Aula virtual: Hablan los usuarios”. [artículo en línea]. Revista

Digital de Investigación en Docencia Universitaria (RIDU) Año 3 -N²-Diciembre 2007. [Fecha de consulta:

dd/mm/aa]. < http://beta.upc.edu.pe/calidadeducativa/ridu/2007/ridu4 6LSpdf>> 


\section{Lea Sulmont Haak}

lsulmont@upc.edu.pe

Doctora en Ciencias de la Educación y Magíster en la especialidad de dispositivos de formación a distancia de la Universidad de Ciencias y Tecnologías de Lille (USTL), Francia

Investigadora, docente y consultora en e-learning. Experiencia en la dirección de proyectos de integración de TICs y de desarrollo de recursos educativos digitales en el ámbito universitario y empresarial. Formadora de formadores en pedagogía y aplicación de tecnologías en la educación.

Actualmente se desempeña como jefe del Área de Tecnologías de la Información y de la Comunicación en la Educación (TICE) del Departamento de Calidad Educativa de la UPC. 